idea has nonetheless been widely accepted by the public and even by scientists who are not familiar with TSEs. I would simply re-iterate that the protein-only theory is still just that: a theory. Several prominent TSE scientists believe that the initial conversion of normal prion protein to an abnormal form is not dependent on protein alone. Perhaps a third Nobel prize in the TSE discipline will be awarded to whoever performs the critical experiments that unequivocally prove or disprove the protein-only theory.

Richard E. Race is at the National Institute of

Health Laboratory for Persistent Viral Diseases,

Rocky Mountain Laboratories, Hamilton,

Montana 59840, USA.

\section{More on prion diseases}

The Pathological Protein: Mad Cow, Chronic Wasting, and Other Deadly Prion Diseases

by Philip Yam

Springer: 2003. $£ 21, \$ 27.50$, E29.95

Copernicus Books: 2003. $\$ 27.50$

\section{$\bullet \bullet \bullet \bullet \bullet \bullet \bullet$ A collection to remember}

\section{Memory From A to $\mathbf{Z}$}

by Yadin Dudai

Oxford University Press: 2002. 338 pp.

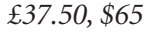

\section{Larry R. Squire}

Like many topics in contemporary neuroscience, the study of memory is being approached using a variety of techniques and at many levels of analysis. Work ranges from the molecular and cellular biology of synaptic change in cell culture and brain slices to the analysis of memory in behaving organisms. Mindful of the diverse backgrounds of those who come to the study of memory, neuroscientist Yadin Dudai has written a charming book designed especially for the student. "This book," he writes, "contains terms that I wish my students to know."

What follows is an engaging, informative and sometimes playful collection of essays, each 2-3 pages long, around the topic of memory, arranged in alphabetical order. The topics do move from A to $\mathrm{Z}$ (from a priori to Zeitgeist), but the book is a personal selection of keywords and starting points for discussion, not an encyclopedia. Many letters have multiple entries (there are 20 for $\mathrm{C}$ and 13 for P, for example); seven letters ( J, K, Q, U, V, X and $\mathrm{Y}$ ) have no entry at all.

Most of the entries are ones that might be expected in a book about memory and neural plasticity ('synapse', 'glutamate', 'calcium', 'CREB', 'hippocampus', 'long-term potentiation', 'declarative memory', 'forgetting' and 'working memory'), but many of them are

\section{Cinema \\ Return of the mutants}

\section{Science fact is the loose basis for the} fantasy in the current spate of movies based on comic-book characters.

Peter Parker was bitten by a genetically modified spider last year to become Spider-Man (in the original 1960s' comic the spider was radioactive, but

that was a different age).

Director Bryan Singer's latest film, $X$-Men 2 (Twentieth Century Fox), is no different, ending with a flowery but accurate description of evolution by punctuated equilibrium. This second adaptation of the popular comic X-Men opened worldwide at the beginning of May.

The X-Men are a band of

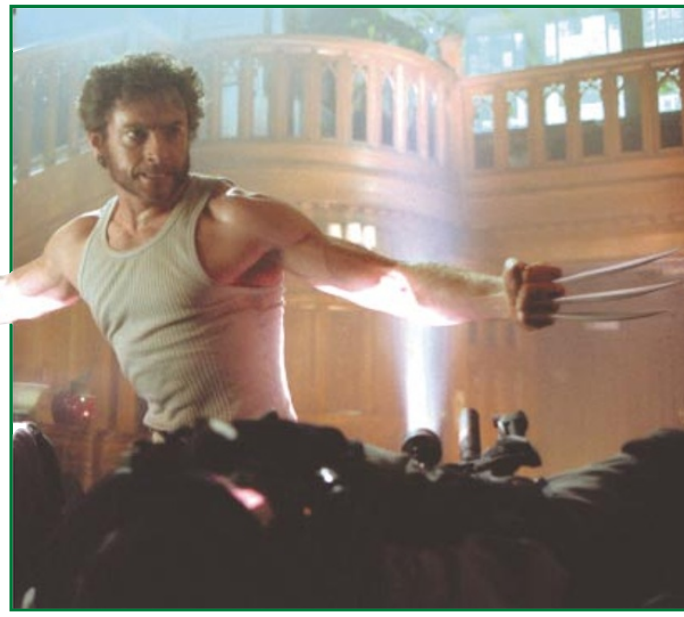
Cutting-edge science? Remarkable powers of regeneration from superheroes (Homo sapiens superior) a mutated $X$ gene make Wolverine a formidable fighting force. who possess a mutated $X$ gene, which has an extraordinarily variable phenotype, allowing some mutants to walk through walls, some to shoot ice from their fingers, and some to perform Moses-like acts of water telekinesis. (The $X$ gene's normal function is not revealed.) The team is led by a powerful telepath, Charles Xavier (played by Patrick Stewart), a benevolent leader who promotes the integration of the mutants into an otherwise hateful society.

Xavier's gang faces two arch-rivals: a human bent on wiping out all of the mutants, and a concentration-camp survivor, Magneto (lan McKellen), who can control magnetic fields to the point of being able to extract iron from

blood. Magneto fervently believes that mutants are the natural inheritors of the Earth and that mankind should voluntarily go extinct to bring this about.

Themes of biological determinism are touched on in this fun, but perhaps overlong, film. Magneto encourages a young mutant to join him, telling him that his genetic abilities render him "a god amongst insects". The wise Xavier offers a counterpoint later: when his team question why one of their number commits an act of self-sacrifice, he weightily declares that the protagonist, against her nature, "made a choice".

Adam Rutherford is the web editor of Nature.

unexpected or even amusing. The entry on 'capacity' provides a thoughtful discussion of what is sometimes called the impossible calculation: how much information-storage capacity does the brain actually have, and does this place any constraint on how much information can be acquired during a lifetime? Entries such as 'artefact', 'controls', 'red herring' and 'Ockham's razor' consider aspects of the scientific method and how science gets done. The entry on 'scoopophobia' (fear of being scooped) presents a humorous discussion about issues of competition in science.

The entry on 'palimpsest' is perhaps a stretch, but it forms the basis for a brief account of how new memory representations form in interactions with previously established ones. There are also separate entries for the organisms that have been important to the scientific study of memory: Aplysia, Drosophila, bird (under 'birdsong'), mouse, rat, monkey and Homo sapiens sapiens.

What makes all this work is that the 131 essays that make up the book are scholarly, up-to-date, even-handed and rich in citations (there are 66 pages of references at the end of the book, along with a full index). Many of the entries begin with an explanation of the classical origins of the terms. Any one of the entries could serve as the basis for classroom discussion and as a starting point for further reading, even for the practising scientist.

A charming feature of the book is that the personal and whimsical list of entries encourages one to try out new ones. For the unused letters, I developed junk DNA, kinase, qualia, quisqualate, ubiquitin, X-rays, Хenopus, vestibulo-ocular reflex, virus and the Yerkes Primate Research Center.

In the end, the book describes an associative network of ideas and facts that, for pedagogical purposes, could be extended and recombined indefinitely. This is an engaging format, and it would be interesting to see it tried with other topics.

Larry R. Squire is at the Veterans Affairs

Healthcare System and the Departments of

Psychiatry, Neurosciences and Psychology,

University of California, San Diego,

California 92161, USA. 